\title{
A SAFE METHOD FOR SECURING ANAEROBIOSIS WITH HYDROGEN. IX *
}

\author{
A. C. Richardion and C. C. DOZleR
}

From the George Williams Hooper Foundation for Medical Research, University of California Medical School. San Francisio

In the course of some quantitative stuclies on the growth of anaerobes in. various plant and animal products, one of the authors (C. C. D.) has, during the past year, employed 13 large anaerobic jars. They have been operated in accordance with the principles proposed by Laidlaw ${ }^{1}$ and constructed according to the descriptions published by McIntosh and Fildes. ${ }^{2}$ It is the purpose of this paper to enumerate briefly the various details and operations which have eliminated the danger of explosions. The arrangement of the equipment as used in this laboratory is shown in fig. 1.

It will be seen from the illustration that the jar is not unlike that described by Brown." A few alterations in construction are indicated in the photograph and are briefly summarized as follows: (1) The wires conveying current to the heating coil are soldered ${ }^{4}$ to short pieces of "Pyrex" glass tubing, which are then cemented into a rubber stopper (A). This method of introducing the lead wires readily permits the use of multiple stranded copper wire (as ordinary, flexible electric light cord), minimizing the chance of breakage. The wires should be looped over and tied to the side of the glass tubes on the outside of the jar top to prevent any strain from occurring at the soldered joint. (2) A shield of asbestos sheet (B) is placed over the upper surface of the coil to prevent the heat from cracking the jar top. (3) Instead of mica as used by McIntosh and Fildes, asbestos washers are placed at these points (C) to deflect the heat from the inner ends of the rubber stoppers.

In several papers, ${ }^{5}$ it is emphasized that the anaerobic apparatus embodying the principle of Brown's jar produces excellent results, but

Received for publication, Aug. 7, 1922.

* This study was aided by a grant from the National Canner's Association, the Canner's League of California and California Olive Association.

\pm Brit. Med. Jour., 1915, 1, p. 497.

2 Lancet, 1916, 1, p. 768.

3our. Exper. Med., 1921, 33, p. 677.

4 Modification of method described by McKelvy (Jour. Am. Chem. Soc. July, 1920). Surface of glass to be soldered coated with metallic platinum by dipping repeatediy in alcoholic $\mathrm{PtCl}_{4}$ solution and heating to a dull red heat.

5 Smillie: Jour. Exper. Med., 1917, 26, p. 59. Berg: Jour. Am. Vet. Assn., 1722, 14. p. 189. Gochenour: Ibid., 1922, 14, p. 301. 
it is also indicated that in all the adaptations or modifications some danger of explosion still persists. The risk of igniting the hydrogen in the jar from the catalyzer itself seems to be eliminated by the use of the wire gauze employed in the construction of the capsule and electrically heated catalyzer described by McIntosh and Fildes. In a recent note, Brown ${ }^{6}$ has, however, pointed out that an explosive mixture still

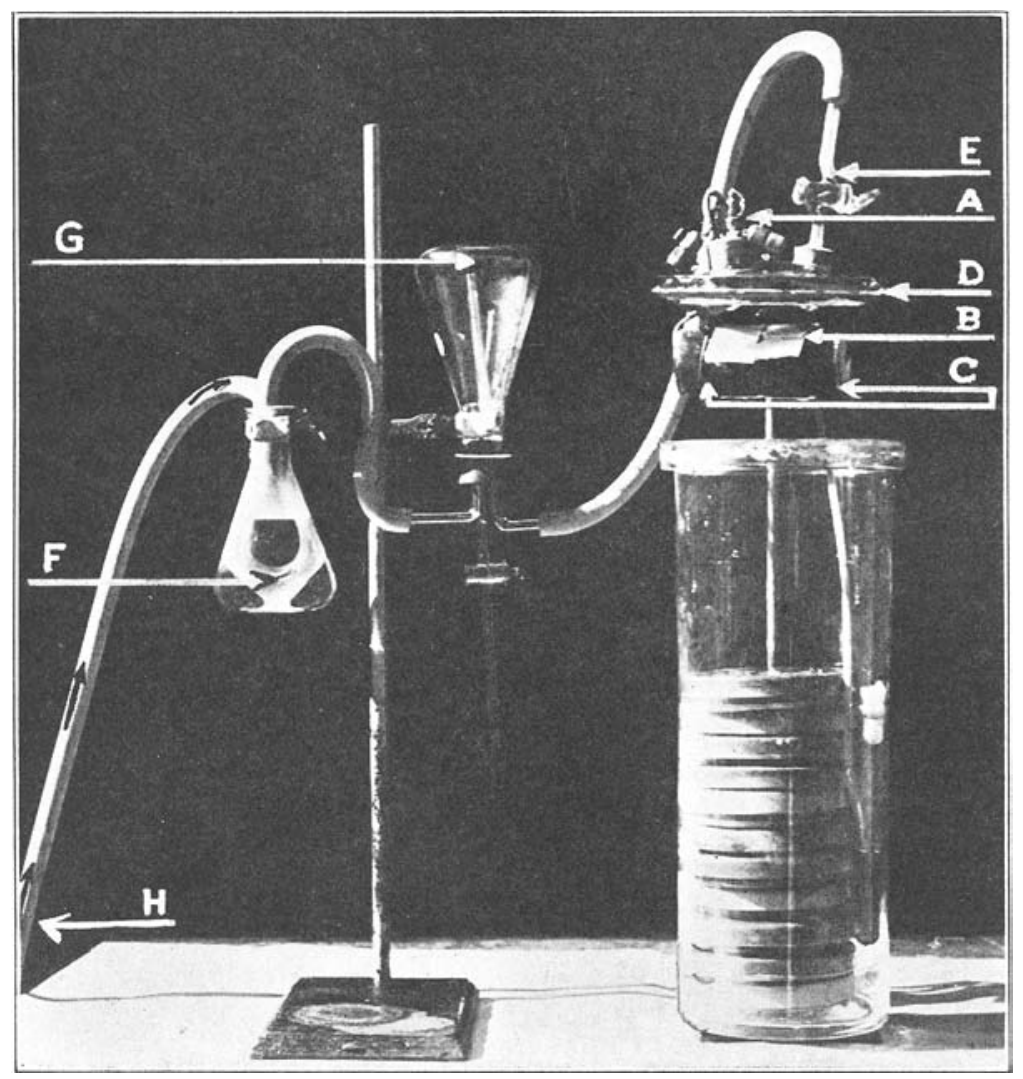

Figure 1

exists in the jar, which may be ignited by a temporary shorting or breaking of the wires, while the circuit is closed. By operating the apparatus in accordance with the method about to be described, the writers believe that the danger of a hydrogen explosion occurring in the jar may be entirely eliminated.

\footnotetext{
B Jour. Exper. Med., 1922, 35, p. 467.
} 


\section{Technic Employed in Operating Jar}

The flange of the jar top (D) is coated to a depth of about $1 / 4$ inch with plasticine modeling clay, which is spread to form a smooth layer of even depth. The top is pressed firmly in the clay and the jar is then evacuated through the stop-cock (E). As a rule, the plasticine joint around the top leaks considerably when the jar is first attached to the vacuum pump, but as soon as the pressure within the jar is reduced, the top will sct more firmly, forcing out a ridge of plasticine. By spreading the projecting clay smoothly over the interstice between the jar and top, a tight seal may readily be obtained. Plasticine exposed to $37 \mathrm{C}$. becomes brittle, and frequent renewal is advocated. When evacuation of the $\mathrm{jar}$ is completed, the stop-cock (E) is closed and the jar connected to the hydrogen supply. Hydrogen is passed in under very slight pressure until the pressure within the jar reaches that of the hydrogen supply. A convenient apparatus for controlling the gas flow is shown ( $F, G$ and $H$ ) and will be described in detail in a subsequent paragraph. An electric current of suitable strength is passed through the heating coil until a satisfactory anaerobiosis is secured.? The jar is permitted to remain connected with the hydrogen supply during this operation, after which the stop-cock is closed and the jar disconnected for incubation.

A Kipp generator will supply hydrogen at sufficient pressure to give satisfactory results with this method. Where hydrogen can be obtained in cylinders, a much more convenient and dependable supply is afforded. A reducing valve is desirable but not absolutely necessary with the gas controlling apparatus shown in fig. 1, which consists of the following parts: (F) flask (open at top) containing a loop of pressure tube submerged in water; a slit about $3 / 4$ inch long in the rubber tube in the flask (F) functions as a safety valve; $(G)$ inverted flask fitted with a three-holed rubber stopper, accommodating a hydrogen intake tube (short), a hydrogen discharge tube (long), and a drain tube (short) with stop-cock. $H$ indicates the hydrogen supply. This apparatus serves as a trap to remove any watcr which may be carried over from the flask $(F)$.

In practice, the "safety-valve" device also serves as a visible index of the gas flow. A rapid flow of gas is permittcd until the vacuum in the jar is overcome, permitting the filling of the jar in a few seconds. When the jar has been filled, as indicated by the emission of gas at the slit, the flow of hydrogen may be reduced (until only a tiny stream of bubbles escapes) during the remainder of the operation.

If the jar is to be incubated much above room temperature, it is advisable to diminish the pressure in the jar, unless a clamp is applied to hold down the tops. The removal of a few culic centimeters of gas with a pump suffices to prevent the top from being displaced by expansion. It is recommended that the use of a clamp to hold the top in position be avoided until it is disconnected from the electric current and the hydrogen supply. The danger incidental to overfilling or ignition is reduced to a minimum when the top is only retained by the viscosity of the plasticine gasket. This fact is strongly emphasized by an experiment in which an explosion has purposely been induced.

2 No experiments have been made to determine what temperature is necessary to activate the catalyzer, nor exactly how much time is needed to deoxygenize completely the jars at the temperature employed. Preliminary tests with illuminating gas have suggested the following heating capacity, which has been used with entire satisfaction: The heating coil consists of 3 feet of nichrome wire (No, $30 \mathrm{~B}$. and S.). It is operated by connecting in series parallel with two 50 watt lamps on a 110 volt a. c. circuit. The current is usually left on about 20 minutes, one of the lamps being cut out 2 or 3 times after starting, to avoid unmecessary heating of the jar and contents. 
It is evident that the operation of the jar described produces at the time the electric current is passed through the coil, a mixture of gases which is so deficient in oxygen as to be practically nonexplosive. This fact has been conclusively demonstrated by the following experiment :

An anaerobic jar similar to that shown in fig. 1 (except that the catalyzing unit was removed) was sealed, exhausted. and filled with hydrogen as described. An attempt was then made to ignite the gas within the jar by passing an electric current (110 volts, a. c.) through a fine steel filament within the jar. The filament fused, breaking the circuit and consequently inducing a spark of considerable intensity, but no explosion resulted. The test was then repeated, using a jar which was poorly exhausted, the internal pressure being about $30 \mathrm{~cm}$. before the hydrogen was introduced. An explosion of considerable violence took place. It must be stated at once, however, that although the top of the jar was thrown upward with great force, the iar itself remained intact, and no shattering of glass occurred. Obviously, if the top had been clamped on the jar, the destructive force of the explosion would have been greatly increased.

Since the absolute safety of this method depends on the removal of a large proportion of the oxygen by evacuation, it appeared advisable to determine whether or not its application would be of value in laboratories equipped with relatively poor vacuum pumps. With this object in view, a jar was evacuated to $30 \mathrm{~cm}$. pressure (which is readily obtainable with an ordinary aspirator, or water pump), and then the partial vacuum was replaced by hydrogen. This operation was repeated, after which the jar was submitted to the ignition test described. No explosion occurred.

It can be stated without any fear of contradiction that the anaerobe jar constructed according to the principles of Fildes and McIntosh ${ }^{8}$ is the most dependable equipment, which produces regularly a satisfactory degree of anaerobiosis. Problems dealing with the quantitative estimation of bacterial growth of B. botulinus, B. tetani, and so forth, in mediums of varying composition and hydrogen-ion concentration have been made accessible by the use of these jars. Excellent growth has been obtained on the surface as well as in the depth of plain liver or milk agar plates. Papers which are in process of preparation indicate that the study of the physiology of sporulation, enzyme production and colony characteristics has been materially enhanced by the apparatus and operations described. In fact, the isolation and purification of delicate anaerobes have been greatly facilitated by the use of sheep blood agar plates in a reliable anaerobic jar.

From the foregoing, it is concluded:

1. That by means of the technic here described, dependable anaerobiosis may easily be secured in jars of the type described, with no

8 Brit. Jour. Exper. Med., 1921, 2, p. 153. 
danger from possible ignition by the electric current employed in activating the catalyzer; and that in the absence of an efficient vacuum pump the safety of the method may be insured by "washing" the jar several times with hydrogen after a partial evacuation.

2. That by dispensing with the use of a clamp to hold the jar top in position, the danger arising from an explosion from any possible cause may be reduced to a minimum.

3. That jars constructed and operated as described are capable of producing anaerobiosis, with a negligible risk to the operator. 